\title{
ON FIELDS WITH FINITE BRAUER GROUPS
}

\author{
IDO EFRAT
}

\begin{abstract}
Let $K$ be a field of characteristic $\neq 2$, let $\operatorname{Br}(K)_{2}$ be the 2primary part of its Brauer group, and let $G_{K}(2)=\operatorname{Gal}(K(2) / K)$ be the maximal pro-2 Galois group of $K$. We show that $\operatorname{Br}(k)_{2}$ is a finite elementary abelian 2 -group $(\mathbb{Z} / 2 \mathbb{Z})^{r}, r \in \mathbb{N}$, if and only if $G_{K}(2)$ is a free pro- 2 product of a closed subgroup $H$ which is generated by involutions and of a free pro- 2 group. Thus, the fixed field of $H$ in $K(2)$ is pythagorean. The rank $r$ is in this case determined by the behaviour of the orderings of $K$. E.g., it is shown that if $r \leq 6$ then $K$ has precisely $r$ orderings, and if $r<\infty$ then $K$ has only finitely many orderings.
\end{abstract}

\section{Introduction.}

It is an open problem in the theory of algebras to characterize the fields $K$ over which there are only finitely many $K$-central (finite dimensional) division algebras. Equivalently, the Brauer group $\operatorname{Br}(K)$ of $K$ should be finite. As an abelian torsion group, $\operatorname{Br}(K)$ is the direct sum of its $p$-primary components $\operatorname{Br}(K)_{p}, p$ prime, so one has in fact to know when is $\operatorname{Br}(K)_{p}$ finite and when is it trivial. Much light is shed on this problem by the following conjecture of Brumer and Rosen $[\mathbf{B R}]$ which states: for each $p$ either

(i) $\operatorname{Br}(K)_{p}=0$;

(ii) $\operatorname{Br}(K)_{p}$ contains a non-trivial divisible subgroup; or

(iii) $p=2$ and $\operatorname{Br}(K)_{2}$ is an elementary abelian 2-group.

This conjecture has been proven in many cases ([BR], [Mer2], [Wu]) notably, Merkurjev proved it under the assumption that char $K \neq p$ and $K$ contains the group $\mu_{p}$ of roots of unity of order $p$ (or more generally, when $\left(K\left(\mu_{p}\right): K\right) \leq 2$; an alternative proof was given by Kahn $\left.[\mathbf{K}]\right)$. Note that when char $K=p, \operatorname{Br}(K)_{p}$ is divisible, so the conjecture is obviously true. When $\mu_{p} \subseteq K$ we also have $\operatorname{Br}(K)_{p}=0$ if and only if $G_{K}(p)$ is a free pro- $p$ group (here $G_{K}(p)=\operatorname{Gal}(K(p) / K)$, where $K(p)$ is the compositum of all finite Galois $p$-extensions of $K$; cf. Lemma 1.1(a) below). Thus, an essential problem is to characterize the fields $K$ for which $\operatorname{Br}(K)_{2} \cong(\mathbb{Z} / 2 \mathbb{Z})^{r}$ for some $r \in \mathbb{N}$. In this paper we characterize these fields in terms of the group $G_{K}(2)$. Recall that a field $K$ is pythagorean if any sum of squares in $K$ is 
a square; equivalently [B1], $G_{K}(2)$ is topologically generated by involutions. We prove:

Main Theorem. The following conditions on a field $K$ of characteristic $\neq 2$ are equivalent:

(a) $\operatorname{Br}(K)_{2}$ is a finite elementary abelian 2-group;

(b) $G_{K}(2)$ is a free pro-2 product $G_{K}(2)=G_{L}(2) *_{2} \hat{F}$, where $L$ is a pythagorean subextension of $K(2) / K$ satisfying $\left(L^{\times}:\left(L^{\times}\right)^{2}\right)<\infty$ and $\hat{F}$ is a free pro-2 group.

If one omits the finiteness requirements in these two statements, then (b) still implies (a). The converse implication may also be true. However, our approach (which yields this converse result in the finite case, as in the main theorem) relies on a "realization" property for reduced quaternionic structures (see $\S 2$ ). This property is conjectured to hold in general, but is presently only known to hold under certain finiteness assumptions (in $[\mathbf{K}]$ Kahn gives several other conditions which are equivalent to $\operatorname{Br}(K)_{2}$ being of exponent 2 , but these conditions do not seem to yield a pythagorean extension as needed).

This stands in an interesting analogy with the following well known property of the character group $C(K)=\operatorname{Hom}\left(G_{K}, \mathbb{Q} / \mathbb{Z}\right)$ of a field $K$ of characteristic $\neq 2$ (with $G_{K}$ denoting the absolute Galois group of $K$ ): $C(K)_{2}$ is an elementary abelian 2-group if and only if $K$ is pythagorean [DD]. In the same spirit, the Brumer-Rosen conjecture is analogous to a result of Whaples [W], asserting that for any field $K$ one of (i)-(iii) above holds also when we replace $\operatorname{Br}(K)$ by $C(K)$.

In the last two sections we study the size of $\operatorname{Coker}\left({ }_{4} \operatorname{Br}(K) \stackrel{2}{\longrightarrow} \operatorname{Br}(K)_{2}\right)$ in general and relate it to the "real arithmetic" of $K$ - in particular, to the theory of fans. It is shown that if this cokernel is finite then $K$ has only finitely many orderings. We also get some more precise information on $K$ when $\operatorname{Br}(K)_{2}$ is small $(\S 5)$.

I thank Bruno Kahn for drawing my attention to the unpublished chapter $[\mathbf{K}]$ of his thesis. I also thank Jack Sonn for several discussions and an anonymous referee for a simplification in Prop. 5.9.

\section{Preliminaries.}

Let $p$ be a prime number and let $K$ be a field of characteristic $\neq p$ containing a primitive root of unity of order $p$. We collect several (mostly well-known) facts relating $\operatorname{Br}(K)_{p}$ to $G_{K}(p)$. For an abelian group $A$ and a positive integer $n$, let ${ }_{n} A=\operatorname{Ker}(A \stackrel{n}{\longrightarrow} A)$. We write $\mu_{p^{n}}$ for the group of all roots of unity of order dividing $p^{n}$ (over the prime field of $K$ ). After fixing a generator of $\mu_{p}$ we may identify $\mu_{p} \cong \mathbb{Z} / p \mathbb{Z}$ as $G_{K}(p)$-modules. The free 
pro- $p$ product of pro- $p$ groups $\Gamma_{1}, \ldots, \Gamma_{m}$ is denoted by $\Gamma_{1} *_{p} \cdots *_{p} \Gamma_{m}$. We will not distinguish between a central simple $K$-algebra and its equivalence class in $\operatorname{Br}(K)$.

Lemma 1.1. Let $K$ be as above.

(a) For each $n, p^{n} \operatorname{Br}(K) \cong H^{2}\left(G_{K}(p), \mu_{p^{n}}\right)$;

(b) Let $L_{1}, \ldots, L_{m}$ be subextensions of $K(p) / K$ such that $G_{K}(p)=G_{L_{1}}(p) *_{p}$ $\cdots *_{p} G_{L_{m}}(p)$. Then $\operatorname{Br}(K)_{p} \cong \operatorname{Br}\left(L_{1}\right)_{p} \oplus \cdots \oplus \operatorname{Br}\left(L_{m}\right)_{p}$ via restriction.

Proof. (a) This is a consequence of the Merkurjev-Suslin theorem ([MerS]; cf. [JW, 1.7]).

(b) For any finite discrete $G_{K}(p)$-module $A$ one has a natural isomorphism

$$
H^{2}\left(G_{K}(p), A\right) \stackrel{\cong}{\longrightarrow} \bigoplus_{i=1}^{m} H^{2}\left(G_{L_{i}}(p), A\right)
$$

[N, Satz 4.1]. Taking $A=\mu_{p^{n}}$, the assertion follows from (a) by passing to direct limits.

We abbreviate $H^{i}(K)=H^{i}\left(G_{K}(p), \mathbb{Z} / p \mathbb{Z}\right)$. Let $X_{K}$ be the set of orderings of $K$.

Lemma 1.2. Let $K$ be as above, let $K \subseteq L \subseteq K(p)$ be a field, and suppose that $G_{K}(p)=G_{L}(p) *_{p} \hat{F}$, with $\hat{F}$ a free pro-p group. Then:

(a) $\operatorname{Br}(K)_{p} \cong \operatorname{Br}(L)_{p}$ via restriction.

(b) If $p=2$ then Res: $X_{L} \rightarrow X_{K}$ is bijective.

Proof. (a) The fixed field $M$ of $\hat{F}$ in $K(p)$ satisfies ${ }_{p} \operatorname{Br}(M) \cong H^{2}(M)=0$ (Lemma 1.1(a)). Hence $\operatorname{Br}(M)_{p}=0$, so we are done by Lemma 1.1(b).

(b) [B1] yields a natural bijection between $X_{K}$ and the conjugacy classes of the involutions in $G_{K}(2)$, and likewise for $L$. As $\hat{F}$ is torsion-free, the assertion follows from the following general group-theoretic results of Herfort and Ribes [HR, Th. A and Th. B'] and Melnikov [Mel, Prop. 4.9] (independently; see also [EH, Lemma 5.4]): If $\Gamma_{1}, \Gamma_{2}$ are closed subgroups of a pro- $p$ group $G$ such that $G=\Gamma_{1} *_{p} \Gamma_{2}$, then:

(i) every element of finite order in $G$ is conjugate to an element of either $\Gamma_{1}$ or $\Gamma_{2}$

(ii) elements of $\Gamma_{1}$ which are conjugate in $G$ are already conjugate in $\Gamma_{1}$.

Finally, let $v$ be a (Krull) valuation on $K$ (and keep assuming that char $K \neq p$ and $\left.\mu_{p} \subseteq K\right)$. We say that $(K, v)$ is $p$-henselian if $v$ has a unique extension to $K(p)$. Denote the residue field of $(K, v)$ by $k$ and its value group by $\Gamma$, and suppose that char $k \neq p$. Let $\left\{\pi_{j}\right\}_{j \in J}$ be elements of 
$K^{\times}$such that the cosets of $v\left(\pi_{j}\right), j \in J$, form a $\mathbb{Z} / p \mathbb{Z}$-linear base of $\Gamma / p \Gamma$. Denote the collection of all subsets of $J$ with precisely $m \in \mathbb{N}$ elements by $J_{m}$. Also, let $(x)$ be the image of $x \in K^{\times}$in $H^{1}(K)$ under the Kummer isomorphism $K^{\times} /\left(K^{\times}\right)^{p} \cong H^{1}(K)$, and let $\langle(x)\rangle$ be the generated subgroup. There is a natural epimorphism $G_{K}(p) \rightarrow G_{k}(p)$ [EH, Lemma 1.1], giving rise to inflation maps Inf: $H^{i}(k) \rightarrow H^{i}(K)$. Parts (a) and (b) of the following lemma are proved by Wadsworth [W, Th. 3.6 and Remark 3.14]. Part (c) follows using Lemma 1.1(a) and the Kummer isomorphism.

Lemma 1.3. In the setup as above and for all $n \in \mathbb{N}$ we have:

$$
H^{n}(K)=\bigoplus_{m=0}^{n} \bigoplus_{\left\{j_{1}, \ldots, j_{m}\right\} \in J_{m}}\left(\operatorname{Inf}\left(H^{n-m}(k)\right) \cup\left\langle\left(\pi_{j_{1}}\right)\right\rangle \cup \cdots \cup\left\langle\left(\pi_{j_{m}}\right)\right\rangle\right) .
$$

(b) For each $0 \leq m \leq n$ and each set $\left\{j_{1}, \ldots, j_{m}\right\} \in J_{m}$, the map $H^{n-m}(k) \longrightarrow H^{n}(K)$, given by $\varphi \mapsto \operatorname{Inf}(\varphi) \cup\left(\pi_{j_{1}}\right) \cup \cdots \cup\left(\pi_{j_{m}}\right)$, is injective.
${ }_{p} \operatorname{Br}(K) \cong{ }_{p} \operatorname{Br}(k) \oplus\left(k^{\times} /\left(k^{\times}\right)^{p}\right)^{J} \oplus(\mathbb{Z} / p \mathbb{Z})^{J_{2}}$.

\section{Quaternionic structures.}

All fields considered from now on will be assumed to have characteristic $\neq 2$. We keep the cohomological notation of $\S 1$, but with $p=2$.

Our proofs make an essential use of the notion of quaternionic structures, as in $[\mathbf{M}]$. We recall that a quaternionic structure is a triple $\langle G, Q, q\rangle$, consisting of an elementary abelian 2 -group $G$ (written multiplicatively) with a distinguished element -1 , a set $Q$ with a distinguished element 0 , and a surjection $q$ : $G \times G \rightarrow Q$, such that for all $x, x^{\prime}, y, y^{\prime} \in G$ :

(1) $q(x, y)=q(y, x)$;

(2) $q(x,-x)=0($ where $-x:=(-1) x)$;

(3) $q(x, y)=q\left(x^{\prime}, y\right)$ if and only if $q\left(x x^{\prime}, y\right)=0$;

(4) if $q(x, y)=q\left(x^{\prime}, y^{\prime}\right)$ then there exists $z \in G$ such that $q(x, y)=q(x, z)$ and $q\left(x^{\prime}, y^{\prime}\right)=q\left(x^{\prime}, z\right)$.

Morphisms and direct products of quaternionic structures are defined in the obvious way (see $[\mathbf{M}]$ for more details and background). Given subgroups $G_{1}, G_{2}$ of $G$, let $\mathcal{Q}_{i}=\left\langle G_{i}, Q_{i}, q_{i}\right\rangle$ be the induced structures, where $Q_{i}=$ $q\left(G_{i} \times G_{i}\right)$ and $q_{i}=\left.q\right|_{G_{i} \times G_{i}}, i=1,2$. A decomposition $G=G_{1} \times G_{2}$ extends to a decomposition $\mathcal{Q}=\mathcal{Q}_{1} \times \mathcal{Q}_{2}$ precisely when the following holds: for any $x_{1}, y_{1} \in G_{1}, x_{2}, y_{2} \in G_{2}$ we have $q\left(x_{1} y_{1}, x_{2} y_{2}\right)=0$ if and only if $q\left(x_{i}, y_{i}\right)=0$, $i=1,2$ [M, Th. 5.8].

To a field $K$ one associates a quaternionic structure

$$
\mathcal{Q}(K)=\left\langle K^{\times} /\left(K^{\times}\right)^{2}, Q_{K}, q_{K}\right\rangle,
$$


where -1 is $-\left(K^{\times}\right)^{2}, Q_{K}$ is the set of all quaternion algebras in ${ }_{2} \operatorname{Br}(K)$, and $q_{K}$ is the usual quaternionic pairing. To a field extension $L / K$ one associates in a natural way a morphism $\operatorname{Res}: \mathcal{Q}(K) \rightarrow \mathcal{Q}(L)$.

The category of quaternionic structures is naturally equivalent to the category of abstract Witt rings $[\mathbf{M}$, Th. 4.5]. We will not make here any real use of the latter notion; however, in considerations involving quaternionic structures we will freely apply some known results which are formulated in the literature in terms of abstract Witt rings. Under the above-mentioned equivalence, $\mathcal{Q}(K)$ corresponds to the Witt ring $W(K)$ of $K$.

A quaternionic structure $\mathcal{Q}=\langle G, Q, q\rangle$ is called:

(i) completely degenerate if $Q=\{0\}$;

(ii) non-degenerate if $q$ is non-degenerate;

(iii) reduced if for all $x \in G, q(x, x)=0$ implies $x=1$ (equivalently, the map $x \mapsto q(x,-1)$ is injective);

(iv) euclidean if $G=\{1,-1\}$ and $|Q|=2$;

(v) non-basic if $G \neq\{1,-1\}$ and there exists $x \in G$ such that $q(x, y) \neq 0$ for all $y \in G \backslash\{1,-x\}$ and such that $q(-x, y) \neq 0$ for all $y \in G \backslash\{1, x\}$;

(vi) finitely generated if $|G|<\infty$.

Remark 2.1. Let $K$ be a field. Then $\mathcal{Q}(K)$ is completely degenerate precisely when $H^{2}(K)=0$ (by [Mer1]), i.e., when $G_{K}(2)$ is a free pro-2 group [S1, I-32, Prop. 21.2 and I-37, Cor. 2]. Also, $\mathcal{Q}(K)$ is reduced if and only if $K$ is pythagorean [M, pp. 89-90]. Finally, $\mathcal{Q}(K)$ is euclidean if and only if $G_{K}(2)$ has (profinite) rank 1 but is not $\mathbb{Z}_{2}$. By the results of [B1], this means that $K$ is a euclidean field (i.e., $G_{K}(2) \cong \mathbb{Z} / 2 \mathbb{Z}$ ).

The following classification theorem [M, Th. 6.23] is of fundamental importance:

Theorem 2.2 (Marshall). Every finitely generated reduced quaternionic structure is a direct product of finitely many quaternionic structures which are either euclidean or both reduced and non-basic.

Following Arason, Elman and Jacob [AEJ], we call a quaternionic structure $\mathcal{Q}$ realizable if for every field $K$ and a decomposition $\mathcal{Q}(K)=\mathcal{Q} \times \mathcal{Q}^{\prime}$ of quaternionic structures there exists a subextension $K \subseteq L \subseteq K(2)$ such that Res: $\mathcal{Q}(K) \rightarrow \mathcal{Q}(L)$ coincides with the projection $\mathcal{Q} \times \mathcal{Q}^{\prime} \rightarrow \mathcal{Q}$. It is unknown whether every quaternionic structure is realizable. Yet, one has:

Proposition 2.3. A quaternionic structure $\mathcal{Q}$ is realizable in each of the following cases:

(a) $\mathcal{Q}$ is non-basic;

(b) $\mathcal{Q}$ is completely degenerate; 
(c) $\mathcal{Q}$ is euclidean.

Proof. (a) This is [AEJ, Th. 4.8].

(b) Consider a decomposition $\mathcal{Q}(K)=\mathcal{Q} \times \mathcal{Q}^{\prime}$ with $\mathcal{Q}=\langle G, Q, q\rangle$ completely degenerate and $\mathcal{Q}^{\prime}=\left\langle G^{\prime}, Q^{\prime}, q^{\prime}\right\rangle$. Let $A, A^{\prime}$ be $\mathbb{F}_{2}$-linear bases of $G, G^{\prime}$, respectively. Denoting the Frattini subgroup by $\Phi$, we have a perfect duality

$$
G_{K}(2) / \Phi\left(G_{K}(2)\right) \times K^{\times} /\left(K^{\times}\right)^{2} \longrightarrow\{ \pm 1\}
$$

given by $(\bar{\sigma}, \bar{a}) \mapsto\langle\bar{\sigma}, \bar{a}\rangle=\sigma(\sqrt{a}) / \sqrt{a}$ for $\sigma \in G_{K}(2)$ and $a \in K^{\times}$(with $\bar{\sigma}$, $\bar{a}$ denoting the corresponding cosets, and with $\sqrt{a}$ being a fixed square root of $a$ ). Choose subsets $\Sigma, \Sigma^{\prime}$ of $G_{K}(2)$ such that the cosets of $\Sigma \cup \Sigma^{\prime}$ form an $\mathbb{F}_{2}$-linear basis of $G_{K}(2) / \Phi\left(G_{K}(2)\right)$ dual to $A \cup A^{\prime}$. Let $L$ be the fixed field of $\Sigma$ in $K(2)$. For any $b \in L^{\times}$the duality yields $a \in K^{\times}$such that $\langle\bar{\sigma}, \bar{a}\rangle=\langle\bar{\sigma}, \bar{b}\rangle$ for all $\sigma \in \Sigma$ and such that $\left\langle\bar{\sigma}^{\prime}, \bar{a}\right\rangle=1$ for all $\sigma^{\prime} \in \Sigma^{\prime}$. Then $a \equiv b \bmod \left(L^{\times}\right)^{2}$ and $(a) \in G$. As $\mathcal{Q}$ is completely degenerate, we conclude that the quaternionic pairing $q_{L}$ is trivial and Res: $\mathcal{Q}(K) \rightarrow \mathcal{Q}(L)$ coincides with the projection $\mathcal{Q}(K) \rightarrow \mathcal{Q}$.

(c) In this case the projection $\mathcal{Q}(K)=\mathcal{Q} \times \mathcal{Q}^{\prime} \rightarrow \mathcal{Q}$ corresponds to a signature on $K$, hence also to an ordering $P \in X_{K}$ [M, pp. 74-75]. We take $L$ to be a euclidean closure of $K$ with respect to $P$ [B1] (alternatively, one can argue along the lines of (b)).

The following is the immediate quaternionic structure analog of $[\mathbf{J W r}$, Th. 3.4 and Remark 3.5]:

Lemma 2.4 (Jacob, Ware). The following conditions on a field $K$ and fields $K \subseteq L_{1}, \ldots, L_{n} \subseteq K(2)$ are equivalent:

(a) $\mathcal{Q}(K)=\mathcal{Q}\left(L_{1}\right) \times \cdots \times \mathcal{Q}\left(L_{n}\right)$ via restriction;

(b) $G_{K}(2)=G_{L_{1}}(2) *_{2} \cdots *_{2} G_{L_{n}}(2)$.

Lemma 2.5. Every quaternionic structure $\mathcal{Q}$ decomposes as $\mathcal{Q}=\mathcal{Q}_{1} \times \mathcal{Q}_{2}$, with $\mathcal{Q}_{1}$ non-degenerate and $\mathcal{Q}_{2}$ completely degenerate.

Proof. Write $\mathcal{Q}=\langle G, Q, q\rangle$ and decompose $G=G_{1} \times G_{2}$, with $G_{2}=\{x \in$ $G \mid \forall y \in G: q(x, y)=0\}$. For any $x_{1}, y_{1} \in G_{1}$ and $x_{2}, y_{2} \in G_{2}$ we have $q\left(x_{1} x_{2}, y_{1} y_{2}\right)=0$ precisely when $q\left(x_{1}, y_{1}\right)=0$. Therefore, by the criterion mentioned earlier, this decomposition of $G$ gives rise in a natural way to a decomposition $\mathcal{Q}=\mathcal{Q}_{1} \times \mathcal{Q}_{2}$ of quaternionic structures with $\mathcal{Q}_{1}$ and $\mathcal{Q}_{2}$ as in the lemma.

Lemma 2.6. The following conditions on a quaternionic structure $\mathcal{Q}=$ $\langle G, Q, q\rangle$ are equivalent:

(a) If $x \in G$ satisfies $q(x, x)=0$ then $q(x, y)=0$ for all $y \in G$; 
(b) $\mathcal{Q}=\mathcal{Q}_{1} \times \mathcal{Q}_{2}$ with $\mathcal{Q}_{1}$ (resp., $\mathcal{Q}_{2}$ ) a reduced (resp., completely degenerate) quaternionic structure.

Proof. (a) $\Rightarrow(\mathrm{b}): \quad$ First decompose $\mathcal{Q}=\mathcal{Q}_{1} \times \mathcal{Q}_{2}$ as in Lemma 2.5 and write $\mathcal{Q}_{i}=\left\langle G_{i}, Q_{i}, q_{i}\right\rangle, i=1,2$. Suppose that $x \in G_{1}$ satisfies $q_{1}(x, x)=0$. Then also $q(x, x)=0$, so by assumption $q(x, y)=0$ for all $y \in G$. In particular, $q_{1}(x, y)=0$ for all $y \in G_{1}$, whence $x=1$. This shows that $\mathcal{Q}_{1}$ is reduced.

(b) $\Rightarrow\left(\right.$ a): Denote again $\mathcal{Q}_{i}=\left\langle G_{i}, Q_{i}, q_{i}\right\rangle, i=1,2$. Let $x, y \in G$ and suppose that $q(x, x)=0$. Decompose $x=x_{1} x_{2}, y=y_{1} y_{2}$, with $x_{1}, y_{1} \in G_{1}, x_{2}, y_{2} \in$ $G_{2}$. By the decomposition criterion mentioned above, $q_{1}\left(x_{1}, x_{1}\right)=0$. Since $\mathcal{Q}_{1}$ is reduced, $x_{1}=1$. As $\mathcal{Q}_{2}$ is completely degenerate, $q_{2}\left(x_{2}, y_{2}\right)=0$. By the decomposition criterion again, $q\left(x_{2}, y_{1}\right)=0$. Therefore $q(x, y)=$ $q\left(x_{2}, y_{1} y_{2}\right)=0$.

\section{Elementary abelian 2-primary Brauer groups.}

We view the cyclic algebra defined by $\chi \in C(K)$ and $b \in K^{\times}$as a bilinear pairing $C(K) \otimes K^{\times} \rightarrow \operatorname{Br}(K), \chi \otimes b \mapsto(\chi, b)$ [S2, Ch. XIV, §1]. In particular, if $\chi=\chi_{a}$ is the character in ${ }_{2} C(K)$ with kernel $G_{K(\sqrt{a})}, a \in K^{\times}$, then $\left(\chi_{a}, b\right)$ is the quaternion algebra $(a, b)$ in ${ }_{2} \operatorname{Br}(K)$.

Theorem 3.1. The following conditions on a field $K$ are equivalent:

(a) $\operatorname{Br}(K)_{2}$ is an elementary abelian 2-group.

(b) If $a \in K^{\times}$satisfies $(a, a)=0$ then $(a, b)=0$ for all $b \in K^{\times}$.

(c) $\mathcal{Q}(K)=\mathcal{Q}_{1} \times \mathcal{Q}_{2}$ with $\mathcal{Q}_{1}$ (resp., $\mathcal{Q}_{2}$ ) a reduced (resp., completely degenerate) quaternionic structure.

(d) $\cup(-1): H^{2}(K) \rightarrow H^{3}(K)$ is injective.

Proof. (a) $\Leftrightarrow(\mathrm{b})$ : By the results of Merkurjev and Suslin [MerS, §16], the cyclic algebra pairing ${ }_{4} C(K) \otimes K^{\times} \rightarrow{ }_{4} \mathrm{Br}(K)$ is surjective. Thus, (a) means that $(2 \chi, b)=0$ for all $\chi \in{ }_{4} C(K)$ and $b \in K^{\times}$. Furthermore, there is an exact sequence

$$
{ }_{4} C(K) \stackrel{2}{\longrightarrow}{ }_{2} C(K) \stackrel{\delta}{\longrightarrow}{ }_{2} \operatorname{Br}(K),
$$

where $\delta\left(\chi_{a}\right)=(a, a)[\mathbf{S 3}, \mathrm{p} .4]$. Thus, as $\chi$ ranges over ${ }_{4} C(K)$, the character $2 \chi$ ranges over all $\chi_{a} \in{ }_{2} C(K)$ with $a \in K^{\times}$satisfying $(a, a)=0$.

(b) $\Leftrightarrow(\mathrm{c})$ : Use Lemma 2.6.

(a) $\Leftrightarrow(\mathrm{d}): \quad[\mathbf{L L T}$, Cor. A4] (and [Mer1]) yields an exact sequence

$$
{ }_{4} \operatorname{Br}(K) \stackrel{2}{\longrightarrow}{ }_{2} \operatorname{Br}(K) \cong H^{2}(K) \stackrel{\cup(-1)}{\longrightarrow} H^{3}(K) .
$$

Other equivalent conditions are given in [K, Th. 2]. In light of Remark 2.1 we get: 
Corollary 3.2. A field $K$ is pythagorean if and only if:

(i) $\operatorname{Br}(K)_{2}$ is an elementary abelian 2-group; and

(ii) the quaternionic pairing $q_{K}: K^{\times} /\left(K^{\times}\right)^{2} \times K^{\times} /\left(K^{\times}\right)^{2} \rightarrow{ }_{2} \operatorname{Br}(K)$ is non-degenerate.

Corollary 3.3. Let $K$ be a field such that $G_{K}(2)=G_{L}(2) *_{2} \hat{F}$, with $K \subseteq L \subseteq K(2)$ a pythagorean field and $\hat{F}$ a free pro-2 group. Then $\operatorname{Br}(K)_{2}$ is an elementary abelian 2-group.

Proof. Let $M$ be the fixed field of $\hat{F}$ in $K(2)$. By Lemma 2.4, $\mathcal{Q}(K) \cong$ $\mathcal{Q}(L) \times \mathcal{Q}(M)$ via restriction. By Remark 2.1, $\mathcal{Q}(L)$ is reduced and $\mathcal{Q}(M)$ is completely degenerate, so we are done by Theorem 3.1.

Proof of the Main Theorem. Assume (a). Theorem 3.1 yields a decomposition $\mathcal{Q}(K)=\mathcal{Q}_{1} \times \mathcal{Q}_{2}$ with $\mathcal{Q}_{1}$ reduced and $\mathcal{Q}_{2}$ completely degenerate. Write $\mathcal{Q}_{1}=\left\langle G_{1}, Q_{1}, q_{1}\right\rangle$. Since the map $G_{1} \rightarrow Q_{1}$ given by $x \mapsto q(x,-1)$ is injective, and since $Q_{1}$ embeds into the finite group ${ }_{2} \operatorname{Br}(K)$, the structure $\mathcal{Q}_{1}$ is finitely generated. By Theorem 2.2 and Proposition 2.3, $\mathcal{Q}_{1}$ is the product of finitely many quaternionic structures which are reduced and realizable. Moreover, $\mathcal{Q}_{2}$ is also realizable. Using Lemma 2.4 we get $K \subseteq L_{1}, \ldots, L_{n}, L_{n+1} \subseteq K(2)$ such that $G_{K}(2)=G_{L_{1}}(2) *_{2} \cdots *_{2} G_{L_{n+1}}(2)$, such that $\mathcal{Q}\left(L_{1}\right), \ldots, \mathcal{Q}\left(L_{n}\right)$ are reduced and $\mathcal{Q}\left(L_{n+1}\right)$ is completely degenerate, and such that $\mathcal{Q}_{1} \cong \mathcal{Q}\left(L_{1}\right) \times \cdots \times \mathcal{Q}\left(L_{n}\right)$. By Remark $2.1, L_{1}, \ldots, L_{n}$, hence also $L=L_{1} \cap \cdots \cap L_{n}$, are pythagorean, and $G_{L_{n+1}}(2)$ is a free pro-2 group. Clearly, $G_{K}(2)=G_{L}(2) *_{2} G_{L_{n+1}}(2)$. Finally, $L^{\times} /\left(L^{\times}\right)^{2} \cong$ $L_{1}^{\times} /\left(L_{1}^{\times}\right)^{2} \times \cdots \times L_{n}^{\times} /\left(L_{n}^{\times}\right)^{2} \cong G_{1}$, whence $\left(L^{\times}:\left(L^{\times}\right)^{2}\right)<\infty$.

Conversely, assume (b). Then $\operatorname{Br}(K)_{2} \cong \operatorname{Br}(L)_{2}$ via restriction (Lemma 1.2(a)). Since the quaternionic pairing $L^{\times} /\left(L^{\times}\right)^{2} \otimes L^{\times} /\left(L^{\times}\right)^{2} \rightarrow{ }_{2} \operatorname{Br}(L)$ is surjective [Mer1] and $\left(L^{\times}:\left(L^{\times}\right)^{2}\right)<\infty$, the group ${ }_{2} \operatorname{Br}(L)$ is finite. But ${ }_{2} \operatorname{Br}(L)=\operatorname{Br}(L)_{2}$ (Corollary 3.2), so $\operatorname{Br}(K)_{2}$ is a finite elementary abelian 2-group.

Remark 3.4. Any elementary abelian 2-group is realizable as $\operatorname{Br}(K)_{2}$ for some pythagorean field $K$. Indeed, let $A$ be any set (considered as a discrete topological space) and let $X$ be its one-point compactification. Since $X$ is Boolean (i.e., Hausdorff, compact and totally disconnected), a construction of Craven $[\mathbf{C}]$ yields a pythagorean field $K$ satisfying the strong approximation property (SAP) and for which $X_{K} \cong X$. By Corollary 3.2, $\operatorname{Br}(K)_{2}={ }_{2} \operatorname{Br}(K) \cong H^{2}(K)$. It follows from [Er, Th. 3 and Lemma 2] that $\operatorname{Br}(K)_{2} \cong\{ \pm 1\}^{A}$. 


\section{The $T$-invariant.}

Even when $\operatorname{Br}(K)_{2}$ is not an elementary abelian 2-group, one may still extract some information regarding the "reduced" structure of $K$ by considering

$$
T(K)=\operatorname{Coker}\left({ }_{4} \operatorname{Br}(K) \stackrel{2}{\longrightarrow}{ }_{2} \operatorname{Br}(K)\right) .
$$

For $a, b \in K^{\times}$let $\overline{(a, b)}$ be the image of the quaternion algebra $(a, b)$ under the natural projection ${ }_{2} \operatorname{Br}(K) \rightarrow T(K)$. By a result of Lam, Leep and Tignol [LLT, Cor. 5.14], $\overline{(a, b)}=0$ if and only if the Pfister form $\langle\langle-a,-b, 1\rangle\rangle$ is 0 in $W(K)$, or equivalently, if and only if $(a) \cup(b) \cup(-1)=0$ in $H^{3}(K)$. Moreover, by [LLT, Cor. A4] again, $T(K) \cong H^{2}(K) \cup\langle(-1)\rangle$ naturally. Note that if $0 \neq a \in K^{2}+K^{2}$ then $\langle\langle-a, 1\rangle\rangle$ is isotropic, hence $\langle\langle-a,-b, 1\rangle\rangle=0$ in $W(K)$ for all $b \in K^{\times}$. Consequently, the quaternionic pairing induces a bilinear map

$$
K^{\times} /\left(\left(K^{2}+K^{2}\right) \backslash\{0\}\right) \otimes K^{\times} /\left(\left(K^{2}+K^{2}\right) \backslash\{0\}\right) \longrightarrow T(K),
$$

which is surjective by [Mer1] (recall that $\left(K^{2}+K^{2}\right) \backslash\{0\}$ is a group with respect to multiplication [L1, Ch. X, Cor. 1.7]). Given a field extension $L / K$, the restriction map of the Brauer groups induces a functorial restriction homomorphism Res : $T(K) \rightarrow T(L)$.

Lemma 4.1. (a) Let $L_{1}, \ldots, L_{m}$ be subextensions of $K(2) / K$ such that $G_{K}(2)=G_{L_{1}}(2) *_{2} \cdots *_{2} G_{L_{m}}(2)$. Then $T(K) \cong T\left(L_{1}\right) \oplus \cdots \oplus T\left(L_{m}\right)$ via restriction.

(b) Suppose that $(K, v)$ is 2-henselian, let $k, J, J_{2}$ be as in $\S 1$, and assume that char $k \neq 2$ and that $-1 \notin K^{2}$. Then:

$$
T(K) \cong T(k) \oplus\left(H^{1}(k) \cup\langle(-1)\rangle\right)^{J} \oplus(\mathbb{Z} / 2 \mathbb{Z})^{J_{2}} .
$$

Proof. (a) Use Lemma 1.1(b).

(b) This follows from Lemma 1.3.

4.2 Remarks. (1) If $\sqrt{-1} \in K$ then $T(K)=H^{2}(K) \cup\langle(-1)\rangle=\{0\}$.

(2) The triviality of $T(K)$ does not imply that $\operatorname{Br}(K)_{2}$ is divisible. For example, let $K=\mathbb{Q}(\sqrt{-1}, t)$, with $t$ transcendental. By $(1), T(K)=\{0\}$. But by a result of Fein, Schacher and Sonn [FSS], $\operatorname{Br}(K)_{2}$ has a direct summand which is generated as an abelian group by elements $x, y_{2}, y_{3}, y_{4}, \ldots$ subject to defining relations $2 x=0, x=2^{i} y_{i}, i=2,3, \ldots$. Here $2 y_{2}-4 y_{3}$ is an involution which is not divisible by 4 . 


\section{Quantitative results.}

In this section we estimate the size of $\operatorname{Br}(K)_{2}$ in case it is an elementary abelian 2-group. We remark however, that in the special case when $K$ is pythagorean with finitely many square classes (or more generally, with finite chain length - see below), one has a rather complete structure theory for $G_{K}(2)$ (see $[\mathbf{J}]$ or $[\mathbf{M i}]$ ) which allows a case-by-case computation of $\operatorname{Br}(K)_{2}$ (by means of Lemma 1.1(b) and Lemma 1.3). To treat the general case, we use instead the theory of fans and the related notion of a strictly pythagorean field. Throughout this section we assume general familiarity with the theory of ordered fields, e.g., as in [L2].

Let $C(X, G)$ be the group of all continuous maps from a topological space $X$ into a discrete group $G$ (when $X=\emptyset$ we set $C(X, G)=\{0\}$ ). Denote the fundamental ideal of $W(K)$ by $I(K)$. The (reduced) stability index st $(K)$ of $K$ is the minimal positive integer $s$ ( $\infty$ if no such integer exists) such that the total signature sgn: $I(K)^{s} \rightarrow C\left(X_{K}, 2^{s} \mathbb{Z}\right)$ is surjective (where $\left.I(K)^{0}=W(K)\right)[\mathbf{L} \mathbf{2}, \S 13]$. For $S \subseteq K$ let $X_{K}(S)=\left\{P \in X_{K} \mid S \subseteq P\right\}$ endowed with the Harrison topology $[$ L2, p. 1].

Recall that a field $E$ is strictly pythagorean if $E^{2}$ is a fan. Equivalently, $E$ is formally real and $E(2)$-hereditarily pythagorean (i.e., all formally real subextensions $E(2) / E$ are pythagorean) [B2, p. 89, Th. 2]. In this case $G_{E}(2) \cong \mathbb{Z}_{2}^{m} \times(\mathbb{Z} / 2 \mathbb{Z})$ for some cardinal number $m$, where the generator of $\mathbb{Z} / 2 \mathbb{Z}$ acts on $\mathbb{Z}_{2}^{m}$ by inversion, and $\left|X_{E}\right|=2^{m}[$ B2, pp. 86-87 and p. 124]. Considering $m$ also as an ordinal number, we may construct such a strictly pythagorean field $E$ as follows: order $\Gamma=\mathbb{Z}^{m}$ lexicographically and let $E$ be the field of formal power series $\sum_{\gamma \in \Gamma} a_{\gamma} t^{\gamma}$, with $a_{\gamma} \in \mathbb{R}$ and with $\left\{\gamma \in \Gamma \mid a_{\gamma} \neq 0\right\}$ well-ordered.

The following lemma (essentially due to Becker) allows one to estimate from below the size of $T(K)$ :

Lemma 5.1. Let $m$ be a positive integer and let $K$ be a field with $\operatorname{st}(K) \geq$ $m$. There exists a strictly pythagorean field $K \subseteq E \subseteq K(2)$ such that:

(a) $E=K E^{2}$;

(b) $\left|X_{E}\right|=2^{m}$;

(c) Res: $X_{E} \rightarrow X_{K}$ is injective;

(d) $T(E)=\operatorname{Br}(E)_{2} \cong(\mathbb{Z} / 2 \mathbb{Z})^{\left(m^{2}+m+2\right) / 2}$;

(e) Res: $T(K) \rightarrow T(E)$ is surjective.

Proof. Since $m$ is positive, $K$ is formally real. As st $(K) \geq m,[\mathbf{L 2}$, Th. 13.7] yields a fan $S_{0}$ on $K$ with $\left|X_{K}\left(S_{0}\right)\right| \geq 2^{m}$. Hence $\left(K^{\times}: S_{0}^{\times}\right) \geq 2^{m+1}$. Take a subgroup of $S$ of $K^{\times}$containing $S_{0}^{\times}$but not -1 such that $\left(K^{\times}: S\right)=2^{m+1}$. By [L2, Th. 5.5(2) and Remark 5.2], $S \cup\{0\}$ is a fan, whence $\left|X_{K}(S)\right|=$ 
$2^{m}$. [B2, p. 143: Th. 7] now yields a strictly pythagorean field $K \subseteq E \subseteq$ $K(2)$ satisfying (a)-(c). Assertion (d) follows from [B2, p. 128: Th. 20]. By [Mer1] (or by [B2, p. 128: Th. 20] again), $\operatorname{Br}(E)_{2}$ is generated by quaternion algebras. Combined with (a), this gives (e).

For each $P \in X_{K}$ fix a euclidean closure $\bar{K}_{P}$ of $K$ with respect to $P$ [B1]. Since $T\left(\bar{K}_{P}\right)=\operatorname{Br}\left(\bar{K}_{P}\right)_{2} \cong \mathbb{Z} / 2 \mathbb{Z}$ (with $(-1,-1)$ as the non-trivial element), we may identify $\prod_{P \in X_{K}} T\left(\bar{K}_{P}\right)$ with the group of all mappings $X_{K} \rightarrow\{ \pm 1\}$. Under this identification, the image of the restriction $T(K) \rightarrow \prod_{P \in X_{K}} T\left(\bar{K}_{P}\right)$ is contained in $C\left(X_{K},\{ \pm 1\}\right)$, by [Mer1] again. In this manner we get an $\mathbb{F}_{2^{-}}$ linear map $\Lambda_{K}: T(K) \rightarrow C\left(X_{K},\{ \pm 1\}\right.$ ) (which is independent of the choice of the euclidean closures).

Recall that the chain length $\mathrm{cl}(K)$ of a pythagorean field $K$ is the supremum of all $n \in \mathbb{N}$ for which there exists a proper chain $H_{K}\left(a_{0}\right) \subset H_{K}\left(a_{1}\right) \subset$ $\cdots \subset H_{K}\left(a_{n}\right)$ of (subbasic) Harrison sets, with $a_{0}, a_{1}, \ldots, a_{n} \in K([\mathbf{L} 2, \S 8]$, [EH, $\S 2])$.

Proposition 5.2. Let $K$ be a field.

(a) If $K$ is pythagorean and $\operatorname{cl}(K)<\infty$ then $\Lambda_{K}$ is injective.

(b) $\Lambda_{K}$ is surjective if and only if $\operatorname{st}(K) \leq 2$.

Proof. (a) As $T(K)={ }_{2} \operatorname{Br}(K)$ (by Corollary 3.2), this follows from Jacob's results in $[\mathbf{J}, \S 5]$. Note that when $K$ is pythagorean and not formally real (a case not covered in $[\mathbf{J}]) \sqrt{-1} \in K$, so $T(K)=0$ by Remark 4.2(1).

(b) [Mer1] gives an epimorphism $I(K)^{2} \rightarrow{ }_{2} \operatorname{Br}(K)$ mapping the Pfister form $\langle\langle-a,-b\rangle\rangle$ to $(a, b)$. We get a natural commutative square:

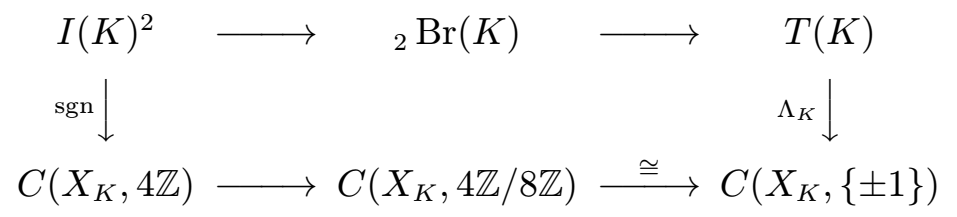

If $\operatorname{st}(K) \leq 2$ then the left vertical map is surjective, whence so is $\Lambda_{K}$.

Conversely, suppose that $\operatorname{st}(K) \geq 3$. Let $E$ be as in Lemma 5.1 with $m=3$. Identifying $X_{E}$ with its image under the injection Res: $X_{E} \rightarrow X_{K}$, we get a commutative square:

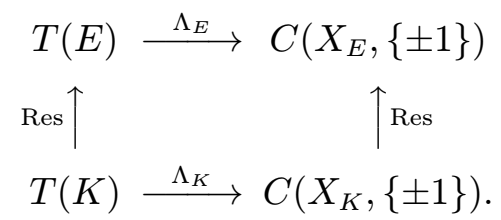

Since $X_{E}$ is finite, the right vertical map is surjective. As $T(E) \cong(\mathbb{Z} / 2 \mathbb{Z})^{7}$ and $C\left(X_{E},\{ \pm 1\}\right) \cong(\mathbb{Z} / 2 \mathbb{Z})^{8}$, the map $\Lambda_{E}$ is not surjective. Therefore $\Lambda_{K}$ is not surjective. 
Corollary 5.3. Let $K$ be a field such that either $\left|X_{K}\right| \leq 7$ or $\operatorname{dim}_{\mathbb{F}_{2}} T(K) \leq$ 6. Then $\left|X_{K}\right| \leq \operatorname{dim}_{\mathbb{F}_{2}} T(K)$.

Proof. In both situations, Lemma 5.1 implies that $s t(K) \leq 2$. By Proposition $5.2(\mathrm{~b}), \Lambda_{K}$ is surjective.

Remark 5.4. In general, $\Lambda_{K}$ need not be injective. For example, let $K$ be a field in which -1 is a sum of 8 , but not less, squares (such fields exist by a result of Pfister $\left[\mathbf{L} 1\right.$, Ch. XI, Th. 2.8]). Then $X_{K}=\emptyset$, but by the observations in $\S 4, \overline{(-1,-1)} \neq 0$ in $T(K)$.

Proposition 5.5. Let $K$ be a field with $\operatorname{Br}(K)_{2} \cong(\mathbb{Z} / 2 \mathbb{Z})^{r}$ and $0 \leq r \leq 6$. Then $\left|X_{K}\right|=r$.

Proof. In light of the main theorem and Lemma 1.2, we may assume that $K$ is pythagorean. By Lemma 5.1 and the assumption, $\operatorname{st}(K) \leq 2$. By Corollary 5.3, $\left|X_{K}\right|<\infty$, hence also $\operatorname{cl}(K)<\infty$. The assertion therefore follows from Proposition 5.2.

Similarly we obtain:

Proposition 5.6. Let $K$ be a pythagorean field with $\left|X_{K}\right| \leq 7$. Then $\operatorname{Br}(K)_{2} \cong(\mathbb{Z} / 2 \mathbb{Z})^{\left|X_{K}\right|}$.

Proposition 5.7. Let $K$ be a field with $\operatorname{Br}(K)_{2} \cong(\mathbb{Z} / 2 \mathbb{Z})^{r}$ and $0 \leq r \leq 3$. Then $G_{K}(2)$ is the free pro- 2 product of $r$ copies of $\mathbb{Z} / 2 \mathbb{Z}$ and of a free pro- 2 group.

Proof. In light of the main theorem and Lemma 1.2(a), we may assume again that $K$ is pythagorean. Lemma 5.1 implies that $\operatorname{st}(K) \leq 1$. Thus $K$ is an SAP field [L2, Cor. 17.11]. By a result of Eršov [Er, Th. 3], $G_{K}(2)$ is then a free pro- 2 product of finitely many copies of $\mathbb{Z} / 2 \mathbb{Z}$. The fixed fields in $K(2)$ of these copies are euclidean, hence have $\mathbb{Z} / 2 \mathbb{Z}$ as their 2-primary Brauer group. Conclude from Lemma 1.1(b) that the number of these copies is $r$, as required.

Remark 5.8. The bounds in Propositions 5.5-5.7 are the best possible. Indeed, let $K$ be a strictly pythagorean field with 8 orderings (see the remarks before Lemma 5.1). Then $\operatorname{Br}(K)_{2} \cong(\mathbb{Z} / 2 \mathbb{Z})^{7}$. Thus, Proposition 5.5 is false for $r=7$ and Proposition 5.6 is false for $\left|X_{K}\right|=8$. When $K$ is a strictly pythagorean field with 4 orderings, $\operatorname{Br}(K)_{2} \cong(\mathbb{Z} / 2 \mathbb{Z})^{4}$ and $G_{K}(2) \cong$ $\mathbb{Z}_{2}^{2} \rtimes(\mathbb{Z} / 2 \mathbb{Z})$, with the generator of $\mathbb{Z} / 2 \mathbb{Z}$ acting on $\mathbb{Z}_{2}^{2}$ by inversion. This Galois group is not a free pro- 2 product of 4 copies of $\mathbb{Z} / 2 \mathbb{Z}$ and of a free pro-2 group (e.g., since the chain length of the former group is 2 and that of the latter is 4; cf. [EH, Lemma 2.1]). Consequently, in Proposition 5.7 one cannot take $r=4$. 
Proposition 5.9. Let $K$ be a field with $T(K)$ finite. Then $X_{K}$ is finite.

Proof. Let $D$ be the kernel of the homomorphism $K^{\times} \rightarrow H^{3}(K)$ given by $a \mapsto(a) \cup(-1) \cup(-1)$. Equivalently, $D$ is the set of all $a \in K^{\times}$which are sums of four squares in $K$ (cf. $\S 4)$. The signature map therefore induces an embedding $X_{K} \hookrightarrow \operatorname{Hom}\left(K^{\times} / D,\{ \pm 1\}\right)$. But

$$
K^{\times} / D \cong H^{1}(K) \cup\langle(-1)\rangle \cup\langle(-1)\rangle \subseteq H^{2}(K) \cup\langle(-1)\rangle \cong T(K) .
$$

Therefore $K^{\times} / D$, whence also $X_{K}$, are finite.

\section{References}

[AEJ] J.K. Arason, R. Elman and B. Jacob, Rigid elements, valuations, and realization of Witt rings, J. Algebra, 110 (1987), 449-467.

[B1] E. Becker, Euklidische Körper und euklidische Hüllen von Körpern, J. reine angew. Math., 268-269 (1974), 41-52.

[B2] E. Becker, Hereditarily-pythagorean Fields and Orderings of higher Level, IMPA Lect. Notes, 29, Rio de Janeiro 1978.

[BR] A. Brumer and M. Rosen, On the size of the Brauer group, Proc. Amer. Math. Soc., 19 (1968), 707-711.

[C] T.C. Craven, Existence of SAP extension fields, Arch. Math., 29 (1977), 594-597.

[DD] J. Diller and A. Dress, Zur Galoistheorie pythagoreischer Körper, Arch. Math., 16 (1965), 148-152.

[EH] I. Efrat and D. Haran, On Galois groups over pythagorean and semi-real closed fields, Isr. J. Math. 85 (1994), 57-78.

[Er] Yu. L. Eršov, Galois groups of maximal 2-extensions, Mat. Zametki, 36 (1984), 913-923 (Russian); Math. Notes (1985), 956-961 (English translation).

[FSS] B. Fein, M. Schacher and J. Sonn, Brauer groups of rational function fields, Bull. Amer. Math. Soc., 5 (1979), 766-768.

[HR] W.N. Herfort and L. Ribes, Torsion elements and centralizers in free products of profinite groups, J. reine angew. Math., 358 (1985), 155-161.

[J] B. Jacob, On the structure of pythagorean fields, J. Algebra, 68 (1981), 247-267.

[JW] B. Jacob and A. Wadsworth, A new construction of noncrossed product algebras, Trans. Amer. Math. Soc., 293 (1986), 693-721.

[JWr] B. Jacob and R. Ware, A recursive description of the maximal pro-2 Galois group via Witt rings, Math. Z., 200 (1989), 379-396.

[K] B. Kahn, Divisibilité du group de Brauer, In: Représentations galoisiennes et classes caractéristiques, Thèse d'Etat, Université de Paris 7, 1987.

[L1] T.Y. Lam, The Algebraic Theory of Quadratic Forms, W.A. Benjamin, Reading, Massachusetts, 1973.

[L2] T.Y. Lam, Orderings, valuations and quadratic forms, Conf. Board of the mathematical sciences, 52, AMS 1983.

[LLT] T.Y. Lam, D.B. Leep and J.-P. Tignol, Biquaternionic algebras and quartic extensions, Publ. Math. IHES, 77 (1993), 63-102. 
[M] M. Marshall, Abstract Witt Rings, Queen's Pap. Pure Appl. Math., 57 (1980).

[Mel] O.V. Melnikov, Subgroups and homologies of free products of profinite groups, Izv. Akad. Nauk SSSR, Ser. Mat. 53 (1989), 97-120 (Russian); Math. USSR Izv., 34 (1990), 97-119 (English translation).

[Mer1] A.S. Merkurjev, On the norm residue symbol of degree 2, Dokl. Akad. Nauk SSSR, 261 (1981), 542-547 (Russian); Sov. Math. Dok., 24 (1981), 546-551 (English translation).

[Mer2] A.S. Merkurjev, Brauer groups of fields, Comm. Algebra, 11 (1983), 2611-2624.

[MerS] A.S. Merkurjev and A.A. Suslin, K-cohomology of Brauer-Severi varieties and the norm residue homomorphism, Izv. Akad. Nauk SSSR, Ser. Mat. 46 (1982), 10111046 (Russian); Math. USSR Izv., 21 (1983), 307-340 (English translation).

[Mi] J. Mináč, Galois groups of some 2-extensions of ordered fields, C.R. Math. Rep. Acad. Sci. Canada, 8 (1986), 103-108.

[N] J. Neukirch, Freie Produkte pro-endlicher Gruppen und ihre Kohomologie, Arch. Math., 22 (1971), 337-357.

[S1] J.-P. Serre, Cohomologie Galoisienne, Lect. Notes Math. 5, Springer 1965.

[S2] J.-P. Serre, Local Fields, Springer, Berlin 1979.

[S3] J.-P. Serre, Topics in Galois Theory, Jones and Barlett, Boston 1992.

[W] A.R. Wadsworth, p-henselian fields: K-theory, Galois cohomology and graded Witt rings, Pac. J. Math., 105 (1983), 473-496.

[Wh] G. Whaples, Algebraic extensions of arbitrary fields, Duke Math. J., 24 (1957), 201-204.

[Wu] T. Würfel, Ein Freiheitskriterium für pro-p-Gruppen mit Anwendungen auf die Struktur der Brauer-Gruppe, Math. Z., 172 (1980), 81-88.

Received March 24, 1995 and revised June 27, 1995. The author was supported by an Alexander von Humboldt fellowship 1992/4.

Departament of Mathematics and Computer Science

Ben Gurion University of the Negev

BE'ER-SHEVA 84105

ISRAEL

E-mail address: efrat@math.bgu.ac.il 